\title{
Descomponiendo el síndrome de déficit atencional en el adulto: hacia un entendimiento de su heterogeneidad pronóstica
}

\author{
ANDREA SLACHEVSKY CH. ${ }^{1,2,3,4}$, CAROLINA PÉREZ J.,6,a, \\ JAIME R. SILVA ${ }^{5, a}$, AMPARO RUIZ-TAGLE ${ }^{4, a}$, ROCÍO MAYOL ${ }^{4, a}$, \\ CARLOS MUÑOZ-NEIRA ${ }^{2, a}$, JAVIER NÚÑEZ-HUASAF ${ }^{4,7}$
}

\section{Prognostic heterogeneity of attention deficit/hyperactivity disorder in adults}

Attention Deficit/Hyperactivity Disorder (ADHD) is a clinical syndrome characterized by an onset in early life. More than $65 \%$ of patients persist with manifestations of ADHD in adulthood. These symptoms may interfere in activities of daily-living, interpersonal relationships and professional and academic achievement. Nevertheless, the observation of an important group of adults with $A D H D$ who do not show significant difficulties in the areas mentioned before puts into evidence the prognostic heterogeneity of this disorder. One of the current, most accepted explanations is the Double-Pathway Model: two double-dissociated deficits (Executive Disorders and Delayed-Reward Processing impairments) are involved in the genesis of $A D H D$, which explains the existence of different behavioral phenotypes. Moreover, personality traits like tenacity or perseverance are associated with higher levels of achievement in adults. On these grounds, we propose the hypothesis that the neurobiological correlate of tenacity/perseverance is a preserved Delayed-Reward Processing capacity, although further studies are needed to verify this idea.

(Rev Med Chile 2012; 140: 379-385).

Key words: Achievement; Adult; Deficit Disorder with Hyperactivity; Executive Function.

$\mathrm{E}$ 1 trastorno por déficit de atención/hiperactividad (TDAH) corresponde a un síndrome clínico, caracterizado por un patrón persistente de desatención y/o hiperactividadimpulsividad de inicio en la infancia, que genera dificultades en más de un ámbito de la vida del sujeto que lo padece. Se ha descrito la persistencia de sintomatología en etapas posteriores del ciclo vital en hasta $65 \%$ de los casos ${ }^{1}$, estimándose una prevalencia global de 5,29\% (IC 95\% = 5,01$5,56)^{2}$. El Manual Diagnóstico y Estadístico de los Trastornos Mentales (DSM-IV) ${ }^{3}$, a diferencia de la Clasificación Internacional de Enfermedades

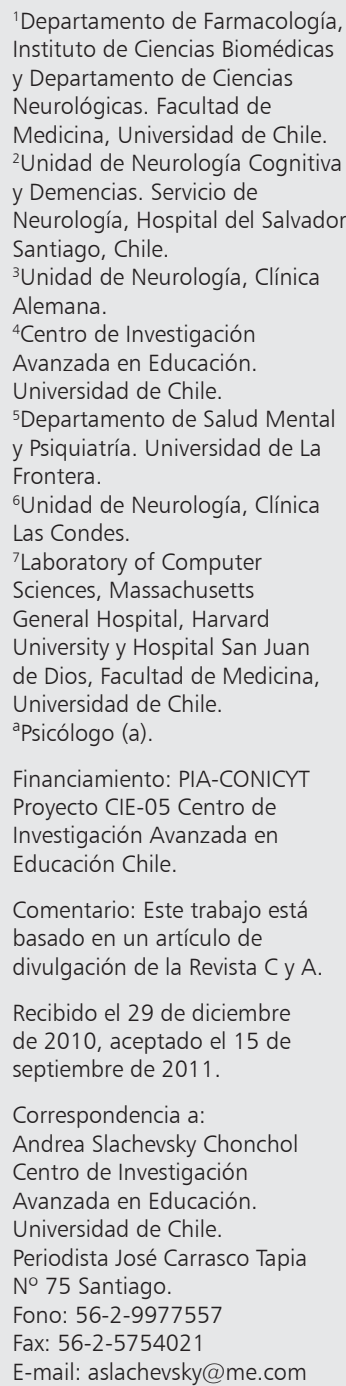


estimado entre 67 a 116 billones de dólares en Estados Unidos de Norteamérica ${ }^{6}$. Un interesante aspecto de esta enfermedad, es la existencia de una gran variabilidad en el impacto del TDAH en las actividades de la vida diaria y en el desempeño laboral ${ }^{7}$. Un grupo significativo de adultos con TDAH logran excelentes resultados en tales actividades. ¿Por qué esta heterogeneidad? ¿Podemos explicarla exclusivamente por la intensidad de los síntomas, la presencia o ausencia de comorbilidad, o el grado de respuesta a los tratamientos? Ciertamente todos estos aspectos inciden, pero esta variabilidad podría estar además relacionada con diferencias en los mecanismos neurobiológicos y la expresión conductual del propio TDAH.

En el presente artículo se aborda la heterogeneidad del TDAH y se sugiere una posible explicación para ella. La perspectiva adoptada se basa en los mecanismos que intervienen en el logro de un comportamiento adaptado y los conocimientos sobre la neurobiología del TDAH. Primero se discutirán los mecanismos cognitivos que intervienen en el logro de una conducta adaptada. Luego, se revisarán los trastornos cognitivos de pacientes afectos de TDAH. Finalmente, se propondrá una hipótesis que puede contribuir a explicar parte de la variabilidad observada en el TDAH. Entender la heterogeneidad del TDAH contribuye a una mejor evaluación clínica de los pacientes, a precisar su impacto y diseñar estrategias terapéuticas más efectivas ${ }^{8}$.

\section{Conducta adaptada y cognición}

Una de las características de la especie humana es su capacidad de representar las vivencias propias como un continuo en el tiempo, desde el pasado y proyectada hacia el futuro. Esa representación estaría posibilitada por la cronoestesia, un tipo de conciencia que permite experimentar el tiempo subjetivo en el que se vive. El ser humano "viaja mentalmente hacia el pasado y también al futuro". La capacidad de representar el futuro explicaría por qué los actos y conductas frecuentemente están dirigidos al logro de objetivos distantes del presente ${ }^{10}$. Incluso, podemos fijarnos objetivos imaginarios no relacionados directamente con las necesidades y situación actual ${ }^{11}$. De este modo una persona es capaz de despreciar recompensas inmediatas, emprendiendo acciones que significan un costo en el presente en pos de esos objetivos futuros. ¿Qué procesos cognitivos intervienen en la realización de esas acciones y en la postergación de la recompensa?. El estudio de pacientes con lesiones cerebrales permite develar los mecanismos cognitivos que hacen posibles los comportamientos adaptados. Desde la publicación de Harlow sobre Phineas Gage en 1868, se han reportado numerosos pacientes con trastornos de la adaptación del comportamiento asociada a lesiones del córtex prefrontal $(\mathrm{CPF})^{12}$. En un grupo de pacientes predominan los cambios de la "personalidad" (impulsividad y pérdida de la iniciativa o motivación) y en otro, de la cognición. En este tipo de afecciones se observa una pérdida del pensamiento abstracto y una incapacidad de planificar y modular una secuencia de acciones considerando las consecuencias futuras de és$\operatorname{tas}^{13,14}$. Tales pacientes presentan también una pérdida de sus "capacidades críticas", es decir, no evalúan su comportamiento ni que tan oportunas son sus acciones. Asimismo, su temporalidad parece limitarse al presente y actuarían en una realidad restringida que no incorpora el contexto ${ }^{15}$. Los elementos anteriores sugieren que estos pacientes estarían en parte desprovistos de cronoestesia, no pudiendo acceder a una representación de sus existencias como un continuo en el tiempo y usar esa información para seleccionar acciones e inhibir respuestas inapropiadas ${ }^{16}$. Las capacidades cognitivas que más intervienen en la adaptación del comportamiento son la planificación, la capacidad de inhibición, el control de interferencias, la resolución de problemas y el razonamiento ${ }^{17}$. $\mathrm{La}$ selección de una acción adecuada a un contexto depende también de componentes emocionales tales como una adecuada motivación, capacidad de tomar decisiones y el procesamiento de las recompensas. Para lograr objetivos y planes de alto nivel, los seres humanos sanos son capaces de controlar deliberadamente el deseo de recompensas inmediatas en pos de recompensas diferidas ${ }^{18}$. En síntesis, la adaptación del comportamiento dependería, entre otros, de dos aspectos esenciales: la capacidad de planificar y la capacidad de diferir las recompensas.

\section{¿Quid del TDAH?}

Se ha propuesto que no existe un único déficit que explique el TDAH, sino que, por el contrario, diferentes trastornos originarían sendos subtipos 
de TDAH: uno en el que predominarían los trastornos cognitivos y otro en el que predominaría un trastorno de la motivación y del procesamiento de la recompensa ${ }^{19}$.

\section{TDAH y funciones ejecutivas}

Los pacientes con TDAH suelen rendir mal en tareas que evalúan las funciones ejecutivas (FE), entendidas como el conjunto de capacidades mentales necesarias para la generación de una secuencia conductual coherente en relación al logro de objetivos predeterminados, asegurando la adaptación del comportamiento a contextos físicos y sociales cambiantes ${ }^{20}$. Los trastornos de estos pacientes se evidencian en tests que evalúan la inhibición de respuestas no pertinentes, la planificación y la memoria de trabajo. Meta-análisis sobre rendimientos de pacientes con TDAH en tests de FE muestran un rendimiento disminuido a nivel de grupo en comparación con sujetos contro$\operatorname{les}^{21}$. Sin embargo, el estudio de trastornos a nivel individual (porcentaje de sujetos con rendimientos disminuidos en los tests) revela que sólo 30 a 50\% de los sujetos rinden mal en éstos. Más aún, sólo existe una correlación débil entre la intensidad de los trastornos en los tests de FE y la intensidad de los síntomas de $\mathrm{TDAH}^{22}$.

La normalidad en el desempeño de los sujetos con TDAH en estas tareas se ha atribuido, a lo menos, a dos posibles causas: i) la baja sensibilidad de los tests para evaluar las disfunciones ejecutivas y ii) la existencia de diferentes subtipos de TDAH. Discutiremos a continuación estas dos posibles explicaciones.

Los tests de evaluación de las FE generalmente utilizados en las evaluaciones neuropsicológicas no siempre reflejan los déficits experimentados por los sujetos ${ }^{21,23}$. En la situación de evaluación se utilizan herramientas altamente estructuradas por el examinador y se entregan numerosas claves ambientales externas, restringiendo el rango de posibles acciones para el sujeto, a diferencia de lo que ocurre en la vida cotidiana, en donde los sujetos se enfrentan a un ambiente en continuo cambio, que requiere priorizar entre múltiples estímulos, seleccionar entre diferentes acciones y monitorear claves ambiguas, en ausencia de reglas que especifiquen cómo llevar a cabo una actividad dada $^{24}$. La discrepancia entre el bajo rendimiento observado en tareas de la vida real y un alto rendimiento en los tests neuropsicológicos se explicaría por las diferentes demandas ejecutivas de ambas situaciones ${ }^{25,26}$. Otra posibilidad es que, más allá de culpar a los métodos de evaluación y pensar que estos buenos resultados corresponden a falsos negativos, efectivamente existan pacientes con TDAH sin trastornos de las FE. En otras palabras, la presencia de disfunción ejecutiva no sería necesaria ni suficiente para explicar todos los casos con $\mathrm{TDAH}^{27,28}$. Varios argumentos apoyan esta hipótesis: i) los trastornos de FE en el TDAH se han asociado a la presencia de comorbilidad y no al TDAH propiamente tal ${ }^{29}$; ii) los tests de FE no siempre diferencian a los pacientes con TDAH de los pacientes con otros trastornos psiquiátricos, como trastornos conductuales, trastorno oposicionista-desafiante, autismo de alto nivel y trastornos de lectura ${ }^{30}$; iii) tampoco diferencian entre subtipos clínicos de $\mathrm{TDAH}^{30}$; y iv) una alta proporción de hermanos asintomáticos de pacientes con TDAH tienen desempeños disminuidos en tests de $\mathrm{FE}^{31}$. Estos resultados conducen a cuestionarse el rol de los trastornos de las FE en la generación de los síntomas del TDAH ${ }^{27,32}$. Algunos estudios sugieren que los trastornos de las FE en el TDAH, se explicarían por dificultades en el procesamiento del lenguaje y de lectura de los pacientes con TDAH $^{33-35}$. Finalmente, los pacientes con TDAH también presentan rendimientos disminuidos en otros dominios cognitivos como la atención (disminución de la velocidad de procesamiento de la información y mayor variabilidad en los tiempos de respuestas en diferentes tipos de tareas). Por lo tanto, esto último reafirma la idea acá sostenida: los trastornos de las FE, por sí solos, no son suficientes para explicar la génesis del $\mathrm{TDAH}^{36}$.

Entonces, ¿Cómo podemos interpretar estos estudios? ¿Existe otro déficit central que explique el TDAH, o es posible reconocer diferentes subtipos de TDAH de acuerdo a los diferentes déficits asociados? Esta última es la hipótesis propuesta por Sonuga-Barke y colaboradores ${ }^{36}$. Indudablemente, un grupo importante de pacientes con TDAH presenta trastornos de las FE, pero también es posible observar la presencia de déficits de distinto tipo, tales como los trastornos en el procesamiento de las recompensas diferidas.

\section{TDAH y el procesamiento de las recompensas}

Junto con los déficits de la esfera cognitiva anteriormente mencionados, cuya manifestación conductual sería presumiblemente fija o estática, se 
ha observado la existencia de un subtipo de TDAH cuya expresión conductual (tanto en términos cuantitativos como cualitativos), sería dinámica; es decir, dependiente del momento y situación particular en la cual un individuo se encuentra ${ }^{37}$ y posiblemente asociada a trastornos en la esfera motivacional ${ }^{38,39}$.

Uno de los fenómenos de este tipo mejor descritos corresponde a la aversión a la espera (AE), que conduce a preferir acciones con una recompensa inmediata, en desmedro de otras que otorgan un beneficio mayor, pero sólo a largo plazo $^{40,41}$. La AE se ha asociado a: 1) la presencia de un afecto negativo en relación a la espera; 2) a un descuento intertemporal (DIT) aumentado (caracterizado por una mayor pérdida del valor subjetivo de una recompensa en relación al tiempo que se debe esperar por ella, en comparación a sujetos controles de similar edad y nivel educacional) y 3 ) impulsividad en la búsqueda de recompensas inmediatas ${ }^{42,43}$. La AE se ha explicado como un trastorno en el procesamiento de recompensas. Mediante neuroimagen funcional, se ha observado que adultos con TDAH presentan, durante la realización de tareas con recompensas inmediatas y diferidas, una hipoactivación en el sistema estriado-ventral (circuito cerebral implicado en la representación de las recompensas). El grado de hipoactivación se correlacionó de forma directa con los síntomas de hiperactividad e impulsivi$\mathrm{dad}^{44}$. Desde otra perspectiva, se ha propuesto que el menor efecto que ejercerían las recompensas diferidas en la modificación de la conducta, puede ser explicado por una alteración en la representación cerebral de la contingencia entre las acciones posibles en el presente y la utilidad asociada a las consecuencias futuras de cada una de ellas. Esto último daría lugar a que aumentos en los tiempos de espera provoquen un mayor descuento en la representación de la utilidad esperada asociada a las recompensas diferidas ${ }^{45}$.

Para estudiar la AE y la sensibilidad a las recompensas normalmente se utilizan tareas de toma de decisión con premios en dinero o en especies de relativa baja cuantía y horizontes temporales de minutos u horas. Estas tareas han demostrado ser de gran utilidad en población infantil, pero de baja sensibilidad para adolescentes y adultos ${ }^{46}$. Esto último se podría explicar por la significativa variación del valor subjetivo del tiempo entre niños y adultos y de la utilidad asociada a los distintos tipos de recompensas. En particular, la valoración de recompensas pequeñas muestra una correlación negativa con la edad, y lo contrario sucede con respecto a los tiempos de espera ${ }^{47,48}$. Sin embargo, a pesar de estas dificultades metodológicas, estudios experimentales han mostrado la existencia de una AE y un trastorno en el procesamiento de la recompensa con tareas similares a las descritas en adultos con TDAH ${ }^{43}$. Para facilitar la evaluación de la AE y trastornos en el DIT, Clare y colaboradores han desarrollado un cuestionario auto-administrado que mostró propiedades psicométricas adecuadas en un primer estudio ${ }^{46}$.

¿Existen subtipos de TDAH? La doble disociación

En múltiples estudios se ha encontrado una doble disociación entre déficits en las capacidades inhibitorias, que son un reflejo de disfunción ejecutiva, y la capacidad de esperar por recompensas diferidas de mayor magnitud ${ }^{40,42,49}$. Esto hace suponer que los déficits ejecutivos y los déficits del procesamiento de las recompensas diferidas (del tipo AE) no serían excluyentes entre sí. De hecho, esta heterogeneidad neuropsicológica del TDAH se apoya en la observación reiterada, en diversos estudios, de cuatro subpoblaciones distinguibles: 1) TDAH con predominio disejecutivo; 2) TDAH con predominio AE; 3) TDAH con ambas disfunciones y 4) un grupo minoritario en que no es posible distinguir ninguna de las alteraciones anteriores ${ }^{28,38,42,50}$. La existencia de este último grupo aún no ha sido explicada, lo que deja abierta la posibilidad de que trastornos neurobiológicos adicionales estén implicados en el origen del $\mathrm{TDAH}^{37}$. De hecho, recientemente se ha descrito en pacientes con TDAH que la representación cerebral de intervalos de tiempo podría constituir un tercer trastorno que podría explicar los trastornos atencionales en estos pacientes ${ }^{36,51}$. Por otro lado, tanto desde la perspectiva biológica como del neurodesarrollo, es plausible la existencia de sinergia entre factores de origen motivacional y otros de origen cognitivo. Se piensa que, durante el desarrollo evolutivo, trastornos del tipo $\mathrm{AE}$ podrían crear condiciones desfavorables para la maduración de las funciones ejecutivas, dado que la presencia de este estilo motivacional limitaría las oportunidades para que un niño consolide las habilidades ejecutivas necesarias para llevar a cabo tareas prolongadas de manera exitosa ${ }^{28}$.

Aparentemente, la clasificación neurobiológica 
aquí propuesta no tendría correlación respecto de los subtipos Inatento, Hiperactivo/Impulsivo y Mixto propuestos en el DSM-IV.

\section{Conclusiones}

¿Por qué algunos pacientes con TDAH tienen historias de fracasos repetidos y otros no? Goethe reflexiona acerca de la importancia de mantenerse en una conducta y de perseverar en ella: "El genio, ese poder que deslumbra a los ojos humanos, no es a menudo otra cosa que perseverancia bien disfrazada." El logro académico se ha relacionado con la capacidad de perseverar y la motivación para cumplir objetivos a largo plazo ${ }^{38,52-54}$. El ser capaz de cumplir una meta futura usualmente requiere mantenerse en actividades rutinarias que no conllevan recompensas inmediatas y que, por el contrario, pueden significar sacrificios importantes en el presente. Esto último sugiere que la perseverancia puede ser un factor tanto o más relevante que la capacidad de planificación por sí sola. En este contexto, es plausible postular al adecuado procesamiento de las recompensas diferidas como el correlato neurobiológico de la perseverancia. Así, la hipótesis de trabajo aquí presentada es que, si bien los pacientes con TDAH y trastornos de las FE presentan dificultades en las actividades de la vida diaria, el subtipo de pacientes con trastornos del procesamiento de las recompensas tendría una incapacidad mayor y no lograrían realizar acciones en pos de metas futuras. En otras palabras, privilegiarían acciones que se traducen en una recompensa inmediata. (No obstante, esta hipótesis requiere ser verificada a través de nuevos estudios).

La pregunta por la relación entre TDAH, FE, procesamiento de las recompensas y los mecanismos generativos del comportamiento adaptado puede conducir a un novedoso entendimiento del TDAH. Asimismo, podría reflejarse en nuevas herramientas para su diagnóstico precoz y el diseño de estrategias terapéuticas más efectivas.

\section{Referencias}

1. Wilens TE, Spencer TJ, Biederman J. A review of the pharmacotherapy of adults with Attention Deficit/hyperactivity disorder. Journal of attention disorders 2002; 5 (4): 189-202.

2. Polanczyk G, de Lima MS, Horta BL, Biederman J,
Rohde LA. The worldwide prevalence of ADHD: a systematic review and metaregression analysis. The American Journal of Psychiatry 2007; 164 (6): 942-8.

3. American Psychiatric Association. Diagnostic and Statistical Manual of Mental Disorders-4th Ed. (DSM-IV-TR) Washington, DC: American Psychiatric Association Press. Inc. 2004.

4. World Health Organization. The ICD-10 Classification of Mental and Behavioural Disorders. Clinical descriptions and diagnostic guidelines. Geneva: 1992.

5. Barkley RA. [Advances in the diagnosis and subtyping of attention deficit hyperactivity disorder: what may lie ahead for DSM-V]. Revista de Neurologia 2009; 48 Suppl 2: S101-6.

6. Biederman J, Faraone SV. The effects of attention-deficit/ hyperactivity disorder on employment and household income. Medscape General Medicine 2006; 8 (3): 12.

7. Stefanatos GA, Baron IS. Attention-deficit/hyperactivity disorder: a neuropsychological perspective towards DSM-V. Neuropsychology Review 2007; 17 (1): 5-38.

8. Sonuga-Barke EJS. On the reorganization of incentive structure to promote delay tolerance: a therapeutic possibility for AD/HD? Neural Plasticity 2004; 11 (1-2): 23-8.

9. Suddendorf T, Addis DR, Corballis MC. Mental time travel and the shaping of the human mind. Philosophical transactions of the Royal Society of London. Series B, Biological Sciences 2009; 364 (1521): 1317-24.

10. Suddendorf T, Corballis MC. The evolution of foresight: What is mental time travel, and is it unique to humans? The Behavioral and Brain Sciences 2007; 30 (3): 299313; discussion 313-351.

11. Brinck I, Gardenfors P. Co-operation and Communication in Apes and Humans. Mind and Language 2003; 18(5): 484-501.

12. Harlow JM. Recovery from the passage of an iron bar through the head. History of Psychiatry 1993; 4 (14): 274-81.

13. Luria A. Higher Cortical Functions in Man. 2nd ed. New York: Springer; 1980.

14. Benton A. Differential behavioral effects in frontal lobe disease. Neuropsychologia 1968; 6 (1): 53-60.

15. Fogelson N, Shah M, Scabini D, Knight RT. Prefrontal cortex is critical for contextual processing: evidence from brain lesions. Brain 2009; 132 (Pt 11): 3002-10.

16. Mesulam M. The human frontal lobes: Transcending the default mode through contingent encoding. In: Stuss D, Knight R, editors. Principles of Frontal Lobe Function. Oxford: Oxford University Press; 2002. p. 8-30.

17. Slachevsky ChA, Pérez JC, Silva CJ, Orellana G, Prenafeta ML, Alegría P, et al. Córtex prefrontal y trastornos del 
comportamiento: Modelos explicativos y métodos de evaluación. Rev Chil Neuro-Psiquiat 2005; 43 (2): 10921.

18. Metcalfe J, Mischel W. A hot/cool-system analysis of delay of gratification: dynamics of willpower. Psychological Review 1999; 106 (1): 3-19.

19. Sonuga-Barke EJS, Sergeant J. The neuroscience of ADHD: multidisciplinary perspectives on a complex developmental disorder. Developmental Science 2005; 8 (2): 103-4.

20. Rabbitt PMA. Methodology of frontal and executive function. Hove, East Sussex: Psychology Press; 1997.

21. Barkley RA, Murphy KR. Impairment in occupational functioning and adult ADHD: the predictive utility of executive function (EF) ratings versus EF tests. Archives of Clinical Neuropsychology 2010; 25 (3): 157-73.

22. Willcutt EG, Doyle AE, Nigg JT, Faraone SV, Pennington BF. Validity of the executive function theory of attention-deficit/hyperactivity disorder: a meta-analytic review. Biological Psychiatry 2005; 57 (11): 1336-46.

23. Levine B, Stuss DT, Milberg WP, Alexander MP, Schwartz M, Macdonald R. The effects of focal and diffuse brain damage on strategy application: evidence from focal lesions, traumatic brain injury and normal aging. Journal of the International Neuropsychological Society 1998; 4 (3): 247-64.

24. Acker M. A review of the Ecological Validity of Neuropsychological Tests. In: Tupper DE, Cicerone KE, editors. The Neuropsychology of Everyday Life: Assessment and Basic Competencies. Volume 2 of Foundations of Neuropsychology. Boston: Springer; 1990. p. 19-56.

25. Shallice T, Burgess PW. Deficits in strategy application following frontal lobe damage in man. Brain 1991; 114 (Pt 2): 727-741.

26. Burgess PW, Alderman N, Forbes C, Costello A, Coates LM-a, Dawson DR, et al. The case for the development and use of "ecologically valid" measures of executive function in experimental and clinical neuropsychology. Journal of the International Neuropsychological Society 2006; 12 (2): 194-209.

27. Willcutt EG, Pennington BF, Olson RK, Chhabildas N, Hulslander J. Neuropsychological analyses of comorbidity between reading disability and attention deficit hyperactivity disorder: in search of the common deficit. Developmental Neuropsychology 2005; 27 (1): 35-78.

28. Sonuga-Barke EJS, Sergeant JA, Nigg J, Willcutt E. Executive dysfunction and delay aversion in attention deficit hyperactivity disorder: nosologic and diagnostic implications. Child and Adolescent Psychiatric Clinics of North America 2008; 17 (2): 367-84, ix.

29. Jonsdottir S, Bouma A, Sergeant JA, Scherder EJA. Re- lationships between neuropsychological measures of executive function and behavioral measures of ADHD symptoms and comorbid behavior. Archives of Clinical Neuropsychology 2006; 21 (5): 383-94.

30. Geurts HM, Verté S, Oosterlaan J, Roeyers H, Sergeant JA. ADHD subtypes: do they differ in their executive functioning profile? Archives of Clinical Neuropsychology 2005; 20 (4): 457-77.

31. Slaats-Willemse DIE, Swaab-Barneveld HJT, de Sonneville LMJ, Buitelaar JK. Family-genetic study of executive functioning in attention-deficit/hyperactivity disorder: Evidence for an endophenotype? Neuropsychology 2007; 21 (6): 751-60.

32. Bidwell LC, Willcutt EG, Defries JC, Pennington BF. Testing for neuropsychological endophenotypes in siblings discordant for attention-deficit/hyperactivity disorder. Biological Psychiatry 2007; 62 (9): 991-8.

33. Bridge Denckla M. ADHD: topic update. Brain \& Development 2003; 25 (6): 383-9.

34. Cohen NJ, Vallance DD, Barwick M, Im N, Menna R, Horodezky NB, et al. The interface between ADHD and language impairment: an examination of language, achievement, and cognitive processing. Journal of Child Psychology and Psychiatry, and Allied Disciplines 2000; 41 (3): 353-62.

35. Jonsdottir S, Bouma A, Sergeant JA, Scherder EJA. The impact of specific language impairment on working memory in children with ADHD combined subtype. Archives of Clinical Neuropsychology 2005; 20 (4): 44356.

36. Sonuga-Barke E, Bitsakou P, Thompson M. Beyond the dual pathway model: evidence for the dissociation of timing, inhibitory, and delay-related impairments in attention-deficit/hyperactivity disorder. Journal of the American Academy of Child and Adolescent Psychiatry 2010; 49 (4): 345-55.

37. Sonuga-Barke EJS, Wiersema JR, van Der Meere JJ, Roeyers H. Context-dependent dynamic processes in attention deficit/hyperactivity disorder: differentiating common and unique effects of state regulation deficits and delay aversion. Neuropsychology review 2010; 20 (1): 86-102.

38. Marco R, Miranda A, Schlotz W, Melia A, Mulligan A, Müller U, et al. Delay and reward choice in ADHD: an experimental test of the role of delay aversion. Neuropsychology 2009; 23 (3): 367-80.

39. Castellanos FX, Sonuga-Barke EJS, Milham MP, Tannock R. Characterizing cognition in ADHD: beyond executive dysfunction. Trends in Cognitive Sciences 2006; 10 (3): 117-23.

40. Bitsakou P, Psychogiou L, Thompson M, Sonuga-Barke 
EJS. Delay Aversion in Attention Deficit/Hyperactivity Disorder: an empirical investigation of the broader phenotype. Neuropsychologia 2009; 47 (2): 446-56.

41. Luman M, Oosterlaan J, Sergeant JA. The impact of reinforcement contingencies on $\mathrm{AD} / \mathrm{HD}$ : a review and theoretical appraisal. Clinical Psychology Review 2005; 25 (2): 183-213.

42. Sonuga-Barke EJS. Causal models of attention-deficit/ hyperactivity disorder: from common simple deficits to multiple developmental pathways. Biological Psychiatry 2005; 57 (11): 1231-8.

43. Scheres A, Lee A, Sumiya M. Temporal reward discounting and ADHD: task and symptom specific effects. Journal of Neural Transmission 2008; 115 (2): 221-6.

44. Ströhle A, Stoy M, Wrase J, Schwarzer S, Schlagenhauf F, Huss M, et al. Reward anticipation and outcomes in adult males with attention-deficit/hyperactivity disorder. NeuroImage 2008; 39 (3): 966-72.

45. Scheres A, Tontsch C, Thoeny AL, Kaczkurkin A. Temporal reward discounting in attention-deficit/hyperactivity disorder: the contribution of symptom domains, reward magnitude, and session length. Biological Psychiatry 2010; 67 (7): 641-8

46. Clare S, Helps S, Sonuga-Barke EJS. The quick delay questionnaire: a measure of delay aversion and discounting in adults. ADHD Attention Deficit and Hyperactivity Disorders 2010; 2 (1): 43-8.

47. Wulfert E, Block JA, Santa Ana E, Rodriguez ML, Colsman M. Delay of gratification: impulsive choices and problem behaviors in early and late adolescence. Journal of personality 2002; 70 (4): 533-52.

48. Green L, Myerson J, Lichtman D, Rosen S, Fry A. Temporal discounting in choice between delayed rewards: the role of age and income. Psychology and Aging 1996; 11 (1): 79-84.

49. Dalen L, Sonuga-Barke EJS, Hall M, Remington B. Inhibitory deficits, delay aversion and preschool $\mathrm{AD} /$ HD: implications for the dual pathway model. Neural Plasticity 2004; 11 (1-2): 1-11.

50. Sonuga-Barke EJS. The dual pathway model of AD/HD: an elaboration of neuro-developmental characteristics. Neuroscience and Biobehavioral Reviews 2003; 27 (7): 593-604.

51. Hwang S-L, Gau SS-F, Hsu W-Y, Wu Y-Y. Deficits in interval timing measured by the dual-task paradigm among children and adolescents with attention-deficit/ hyperactivity disorder. Journal of Child Psychology and Psychiatry, and Allied Disciplines 2010; 51 (3): 223-32.

52. Duckworth AL, Peterson C, Matthews MD, Kelly DR. Grit: perseverance and passion for long-term goals. Journal of Personality and Social Psychology 2007; 92 (6): 1087-101.

53. Duckworth AL, Quinn PD. Development and validation of the short grit scale (grit-s). Journal of Personality Assessment 2009; 91 (2): 166-74.

54. Raizada RDS, Kishiyama MM. Effects of socioeconomic status on brain development, and how cognitive neuroscience may contribute to levelling the playing field. Frontiers in Human Neuroscience 2010; 4 (February): 3. 